\title{
A Focal Zone of Thalamic Plasticity
}

\author{
Jayson L. Parker, ${ }^{1,3}$ Michael L. Wood, ${ }^{2}$ and Jonathan O. Dostrovsky ${ }^{1}$ \\ Departments of ${ }^{1}$ Physiology and ${ }^{2}$ Medical Imaging, University of Toronto, Toronto, Ontario, Canada M5S-1A8, and \\ ${ }^{3}$ Cognitive Neurology Unit, Sunnybrook Hospital, Toronto, Ontario, Canada M4N-3M5
}

In this study, sensory maps in the thalamus were investigated by examining their volume and shape. We determined the forelimb representation in adult rats after the removal of hindlimb input by nucleus gracilis lesions. Three-dimensional reconstructions of thalamic sensory maps were obtained from a grid of electrode penetrations. We found that the volume of the shoulder sensory map contracted $>50 \%$ at an acute time interval $(n=6)$, followed by a robust volumetric sensory map expansion of $25 \%$ at 1 week $(n=8)$ and 1 month $(n=8)$ after lesion relative to controls $(n=8)$. The topology of the volumetric increase was scrutinized by slicing functional maps in the coronal, sagittal, and horizontal planes. The equivalence of such slices from each animal was established by virtue of their distance from either a functional or neuroanatomical landmark.

Plasticity of sensory maps in adult animals was first reported at the thalamic level (Nakahama et al., 1966; Wall and Egger, 1971; Fadiga et al., 1978; Pollin and Albe-Fessard, 1979). Plasticity of somatotopic maps has subsequently been discovered at other levels of the neuraxis in adult animals. With respect to cutaneous pathways, plasticity in somatotopic maps has been demonstrated in the dorsal horn (Basbaum and Wall, 1976; Devor and Wall, 1978), dorsal column nuclei (Dostrovsky et al., 1976; Millar et al., 1976), cerebral cortex (Kalaska and Pomeranz, 1979; Merzenich et al., 1983), and the ventroposteromedial nucleus (Rhoades et al., 1987; Nicolelis et al., 1993). The principle that has emerged from this research is that once a brain region is denervated, adjacent representations expand into the deafferented zone.

In our view, thalamic sensory map plasticity is difficult to detect reliably and has remained polemical. A report of thalamic plasticity by Wall and Egger (1971) has been questioned, in part because of the sparse data presentation given in the paper (Snow and Wilson, 1991; Jaine et al., 1995) and the small magnitude of the forelimb border shift relative to the spacing of electrode penetrations.

Various laboratories have investigated thalamic plasticity after either deafferentation or the reversible blockade of a receptive field and have examined the consequences with a manifold of dependent measures (Nakahama et al., 1966; Fadiga et al., 1978; Pollin and Albe-Fessard, 1979; Garraghty and Kaas, 1991; Shin et al., 1995; Alloway and Aaron, 1996; Rasmusson, 1996a). Com-

Received June 18, 1997; revised Oct. 21, 1997; accepted Oct. 23, 1997.

This research was supported by grants from National Institutes of Health and National Science and Engineering Research Council of Canada. We appreciate the helpful comments on this manuscript by Dr. Rasmusson. We also thank Karen Ma for technical assistance.

Correspondence should be addressed to Jonathan O. Dostrovsky, Department of Physiology, University of Toronto, Medical Sciences Building, Room 3305, Toronto, Ontario, Canada M5S-1A8.

Copyright (C) 1997 Society for Neuroscience $\quad 0270-6474 / 97 / 180548-11 \$ 05.00 / 0$
Surprisingly, all of the volumetric increase unequivocally occurred in a circumscribed coronal slice $300 \mu \mathrm{m}$ thick. This focal zone was located toward the rostral pole of the thalamic tactile relay, the ventroposterolateral nucleus. Analysis in the sagittal plane revealed that, unexpectedly, the shoulder map volume expanded by superimposing its representation on that of the forepaw, via an advancement of the shoulder representation by $0.6 \mathrm{~mm}$ medially. We propose a "hot spot" hypothesis in which focal zones of plasticity may not be specific to the thalamus but may have manifestations elsewhere in the nervous system, such as the cerebral cortex or dorsal column nuclei.

Key words: hot spot; plasticity; thalamus; receptive field; VPL; somatotopy; lemniscal; nucleus gracilis; visualization; topographic overlap; focal zone parisons of map border relationships between control and lesioned animals have been difficult, given the three-dimensional (3-D) character of thalamic sensory maps (Pollin and AlbeFessard, 1979). The proportion of neurons reported to respond to new input after blockade of their original receptive field has ranged from 15 to $70 \%$ (Nakahama et al., 1966; Fadiga et al., 1978; Shin et al., 1995; Alloway and Aaron, 1996). Such variation makes it difficult to use this plasticity as a baseline in which to test hypotheses relating to its mechanism or role in nervous system function.

In this study we have used a protocol that quantifies thalamic plasticity based on a single measure: the volume of somatosensory thalamus responsive to a given body region. It is assumed that increased volume of a thalamic sensory map corresponding to a specific body region, in lesioned animals relative to controls, reflects a change in the receptive field of the neurons sampled. Following the method of Wall and Egger (1971), hindlimb input was removed with lesions of nucleus gracilis. Histology was used to verify electrode placements and to obtain length estimates of certain thalamic nuclei. All volumetric estimates and cross-sectional analysis were performed on a computer displaying the three-dimensional distribution of recording sites for each body region throughout the ventroposterolateral nucleus (VPL).

We asked whether the forelimb, composed of shoulder and forepaw sensory maps, would increase in volume after nucleus gracilis lesions compared with intact controls at various times after lesion. Second, we asked whether our 3-D reconstructions of thalamic sensory maps would reveal any characteristic topological feature behind the volumetric expansion of the forelimb representation. Comparisons of shape were obtained by sectioning functional maps in each plane and comparing the profile of their cross-sectional area. 
A preliminary form of these results has been presented (Parker and Dostrovsky, 1996).

\section{MATERIALS AND METHODS}

Subjects and anesthesia. Thirty male Wistar rats with weights ranging from 250 to $350 \mathrm{gm}$ were used. Four groups of rats were mapped at various times after nucleus gracilis lesions: unoperated controls $(n=8)$, acute group $(n=6), 1$ week after lesion $(n=8)$, and 1 month after lesion $(n=8)$. Rats in the acute group were mapped immediately after their gracile lesion, without recovery from anesthesia. Because mapping takes at least $12 \mathrm{hr}$ to complete, our acute condition covers this span of time immediately after gracile lesions.

Nucleus gracilis lesions. Rats were anesthetized with intraperitoneal ketamine hydrochloride xylazine. The rat's head was tilted forward in the stereotaxic frame, and the obex was exposed surgically. Under a dissecting microscope, lesions were made of the gracile nucleus with jeweler's forceps. Pilot work had established a clear correspondence between the morphological boundary separating nucleus cuneatus and gracilis, with the perimeter of the hindlimb responsive zone as delineated by multiunit recordings. The nucleus was completely macerated with jeweler's forceps down its length. Animals appeared fully recovered from this procedure within $1 \mathrm{hr}$. At no time after lesion was discomfort observed.

Surgery. Animals were water-deprived overnight before mapping of the thalamus. Five minutes before anesthesia, atropine sulfate $(300 \mathrm{mg} / \mathrm{kg}$, s.c.) was injected. Five minutes later, ketamine hydrochloride $(200 \mathrm{mg} /$ $\mathrm{kg}$, i.p.) and xylazine $(50 \mathrm{mg} / \mathrm{kg}$, i.p. $)$ were administered. Maintenance doses of ketamine hydrochloride and xylazine were administered every hour at one-fourth the size of the respective loading dose for the animal. Anesthetic level was maintained by ensuring that there was no response to tail pinch while gentle touch to the eye yielded a weak corneal reflex. Atropine sulfate was administered every $2 \mathrm{hr}(300 \mathrm{mg} / \mathrm{kg}$, s.c.). Body temperature was monitored with a rectal thermometer and kept at $\sim 37^{\circ} \mathrm{C}$ by a thermostatically controlled heating pad underneath the animal. A hole in the trachea was cut, and periodic cleaning with paper tissue followed for at least $20 \mathrm{~min}$ until secretions stopped accumulating. A polyethylene tube was then inserted into the trachea. A unilateral craniotomy contralateral to the side of the gracile lesion was made, extending to all suture lines and the rhinal fissure. The dura was subsequently removed with forceps. Throughout the experiment, the exposed cortex was covered in mineral oil.

Recordings. Multiunit recordings were conducted with glass-coated tungsten microelectrodes with a tip diameter of $35 \mu \mathrm{m}$, possessing an impedance between 2.5 and $3.0 \mathrm{M} \Omega$ at $1000 \mathrm{~Hz}$. Low- and high-pass filters with cutoff frequencies of 6000 and $300 \mathrm{~Hz}$, respectively, were used. The amplifiers, filters, and oscilloscope had the same settings across recording sessions for every animal. The initial electrode penetrations of each session were performed at anteroposterior $-0.35 \mathrm{~cm}$ and mediolateral $\pm 0.33 \mathrm{~cm}$ corresponding to the VPL (Paxinos and Watson, 1997). The electrode was lowered $0.45 \mathrm{~cm}$ relative to the surface of the cortex. Normally, two or three penetrations were required to locate sites responsive to stimulation of the forelimb or hindlimb (and subsequently confirmed histologically). Mapping was performed by making successive penetrations in a two-dimensional grid of electrode penetrations in the rostrocaudal and mediolateral planes in $0.2 \mathrm{~mm}$ increments, with successive recordings in the dorsoventral plane in the same increments. To reduce the mapping time, a restricted number of body regions was examined. For each recording site (Fig. 1) we tested whether the units discharged after the application of brush stimuli to the forepaw (from elbow to paw), shoulder (from elbow to upper extremity of the arm), or hindlimb (thigh or foot). We use the term forelimb to refer to forepaw and shoulder body regions. If a site failed to respond to stimulation of one of these body regions, we classified it as blank. If a site responded to more than one body region, each one was indicated. At the end of the mapping session, two electrolytic lesions were placed (one caudal and one anterior, at the same depth) and the coordinates recorded. The rat was then killed by anesthetic overdose.

Mapping rules. We developed a mapping protocol that produced consistent sensory map data across pilot animals with respect to volume and shape. The rules were (1) successive penetrations along the mediolateral axis were terminated with two contiguous blank penetrations; (2) successive rows of penetrations along the rostrocaudal axis were terminated if an additional row of tracks was blank at each recording site, including one additional penetration medially and laterally for each track; (3) recording commenced in a new track $0.6 \mathrm{~mm}$ above the most dorsal positively identified site obtained in any adjacent electrode track; and (4)
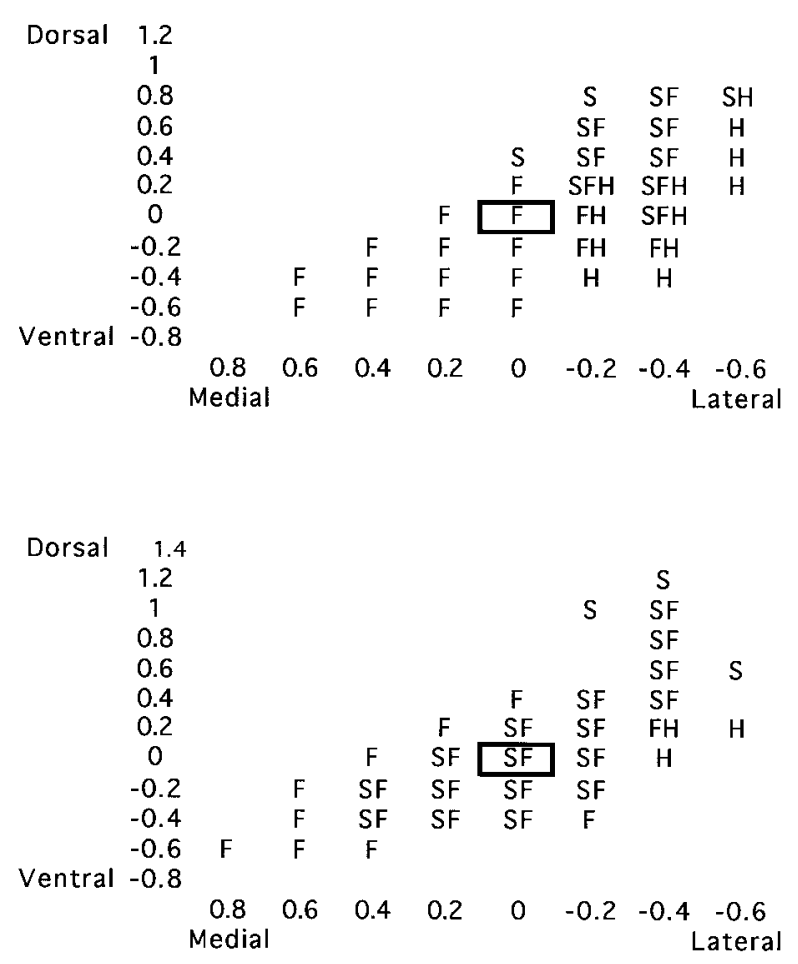

Figure 1. Sample data from control (top) and lesioned (bottom) animals mapped with vertical electrode penetrations. This is a coronal view of a functional map, depicting classifications of recording sites for all body regions examined ( $H$, hindlimb; $F$, forepaw; $S$, shoulder). The forepaw centroid in this plane is the cell with a bold border. For clarity, "blank" recording site designations are omitted.

if no units were encountered within an electrode track, the penetration continued until it was $0.6 \mathrm{~mm}$ below the most ventral positively identified site in any adjacent electrode track. Such rules generated an aggregate map in the VPL representing all three body regions examined that required $80-100$ penetrations per animal and contained $\sim 400$ positively identified sites per animal. On average, from tracheotomy to perfusion, the procedure took $12-14$ hr per animal.

Sensory maps. The forepaw sensory map includes any site responsive to forepaw stimulation, regardless of whether the same site was also responsive to other body regions (Fig. 1). Likewise, the shoulder sensory map includes all sites responsive to shoulder stimulation, ignoring the fact that some sites may have been responsive to other body regions. However, the zone of overlap between shoulder and forepaw includes those sites responsive to both shoulder and forepaw stimulation, regardless of its responsiveness to the stimulation of other body regions.

Histology. Animals were perfused transcardially with saline followed by $4 \%$ paraformaldehyde. Brains were removed, placed in a $30 \%$ sucrose solution of $4 \%$ paraformaldehyde, and sectioned in the horizontal plane with a slice thickness of $0.1 \mathrm{~mm}$. This plane of sectioning allowed us to discern the entire pattern of electrode tracks. Dorsal column nuclei were sectioned coronally with a slice thickness of $40 \mu \mathrm{m}$. All tissue was Nissl-stained.

Volumetric and spatial analysis. Sensory maps were rendered and visualized with Interactive Data Language (Boulder, CO) on a Sun Ultra 170 Sparcstation (Sun Microsystems, Mountain View, CA) from data recorded onto a 3-D spreadsheet. The volume of each sensory map was calculated from the number of recording sites obtained per body region multiplied by the volume element $\left(0.008 \mathrm{~mm}^{3}\right)$ of the grid. Volumetric analysis allowed us to ascertain which body representations underwent expansion. We then conducted spatial analysis on these sensory maps to explore for any characteristic topological features mediating the volumetric changes. Profiles of successive cross-sectional areas of the maps in the coronal, sagittal, and horizontal planes were compared between groups. Equivalent slices of a given sensory map across animals were identified by virtue of their distance from neuroanatomical or functional landmarks. The neuroanatomical landmark was the midpoint of the 
Figure 2. Volume of three thalamic sensory maps at different times after nucleus gracilis lesions. A different group of animals was used for each time point. The recording sites included in the calculation of each sensory map are depicted. Asterisks indicate significant differences between a given treatment group compared with controls. Columns indicate mean; error bars indicate \pm SE.

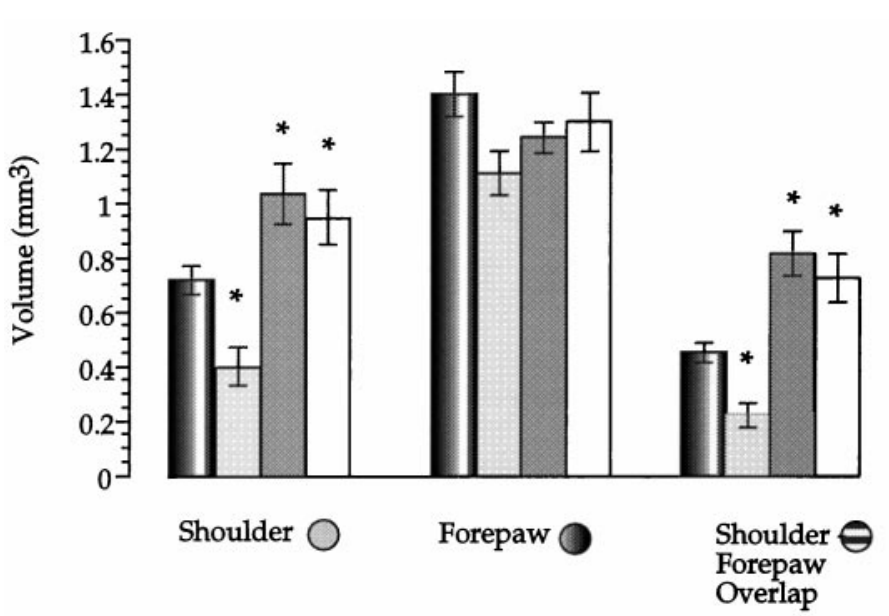

length of the ventrobasal complex (VB) along the anteroposterior axis The functional landmark was the centroid of the sensory map image. For a single axis, the centroid is simply the average coordinate of recording sites (nonblank) along that axis. In 3-D space the centroid is a vector with $x, y$, and $z$ coordinates. For reasons that will become clear in Results, we relied on the centroid reference point for most of our spatial analysis.

Statistics. If the requirements of normality and homogeneity of variance were unsatisfied for a given ANOVA, a correction was performed to the number degrees of freedom with Huynh-Feldt $\epsilon$, making the required level of significance more stringent. All statistics were performed with SPSS version 6.1 for Macintosh (SPSS Inc., Chicago, IL). Post hoc unpaired $t$ test (two-tailed) comparisons that followed ANOVA models are reported as being less than or greater than a 0.05 significance level.

\section{RESULTS}

Volumetric analysis of each sensory compartment is reported first. Sensory maps that exhibited volumetric changes were analyzed in the topology of their expansion. In our topological analysis, we also examined the decrease of hindlimb input in detail.

\section{Volumetric analysis}

Three thalamic sensory map volumes are depicted in Figure 2. The shoulder-forepaw representational overlap refers to any recording site that responded to brush stimuli of both shoulder and forepaw. For each sensory map, all treatment groups are included. An overall multivariate ANOVA of all three sensory map volumes (Fig. 2) revealed a main effect of group (Pillias $_{(9,78)}=$ $0.99 ; p<0.001)$. We then examined the effect of group on each individual sensory map volume with a one-factor ANOVA.

The shoulder sensory map showed an initial contraction in volume relative to controls, followed by a robust volumetric expansion at 1 week and 1 month after lesion. An ANOVA on shoulder sensory map volumes by group revealed a clear treatment effect of lesion $\left(F_{(3,29)}=9.78 ; p<0.001\right)$. Post hoc unpaired $t$ test comparisons indicated that acute and 1 week and 1 month postlesion groups all had significantly different volumes compared with controls (all $p<0.05$ ). Post hoc $t$ test comparisons did not reveal any significant difference between 1 week and 1 month postlesion shoulder map volumes $(p>0.05)$.

The data for forepaw sensory map volumes suggest some contraction after lesions of the gracile nucleus. However, this trend was not statistically significant (ANOVA, $F_{(3,29)}=2.03 ; p>0.1$ ). Therefore, the forepaw sensory map did not show any significant change in volume as a function of time after lesion.

The pattern of results suggested by the volume of representa- tional overlap between the forepaw and shoulder sensory maps appeared to mirror the time course of the shoulder sensory map volumetric changes. Accordingly, an ANOVA revealed a main effect of group $\left(F_{(3,29)}=15.1 ; p<0.001\right)$ on representational overlap. Post hoc $t$ test comparisons indicated that 1 week and 1 month postlesion groups had significantly elevated volumes compared with controls, whereas the acute group significantly decreased in volume relative to controls (post hoc $t$ test). Again, post hoc $t$ tests did not show any significant difference between the volumes obtained at 1 week and 1 month after lesion.

In summary, the shoulder sensory map volume, after an initial period of contraction at an acute time interval, expanded in a robust and consistent manner at 1 week and 1 month after lesion. The volume of sensory map overlap between forepaw and shoulder displayed the same time-dependent changes as the shoulder sensory map after nucleus gracilis lesions. These findings suggest that the shoulder may be increasing its volume by expanding medially and overlapping with the forepaw representation.

\section{Topological analysis of volumetric changes}

We examined the cross-sectional profile of the shoulder sensory map in the coronal, sagittal, and horizontal planes. Along the rostrocaudal axis we discovered a circumscribed region in which all coronal cross-sectional area increases occurred and designated this region a "focal zone." Subsequent analysis in sagittal and horizontal planes focused on this region of interest. Data from the coronal plane were used to compare two different forms of slice alignment from sensory maps: neuroanatomical and functional.

We asked whether the volumetric increase in the shoulder sensory map reflected uniform increases in the cross-sectional area of the map along the anteroposterior axis (Fig. $3 A$ ). Because it was not always possible to discern the border between the ventroposteromedial nucleus (VPM) and the VPL by histology, we used the boundaries of the VB (which includes VPM and VPL) for anatomical measurements. Anatomically, VPM and VPL occupy similar rostrocaudal borders. Each coronal slice from the functional map of a rat is referenced based on its distance from the VB midpoint. In horizontal histological sections the midpoint of $\mathrm{VB}$ was identified between the most rostral border of VB with the reticuler nucleus and caudally with its border against the superior thalamic radiation (Paxinos and Watson, 1997, their Fig. 100). On the abscissa, zero refers to the midpoint of VB. Negative spatial offsets are caudal to the VB 

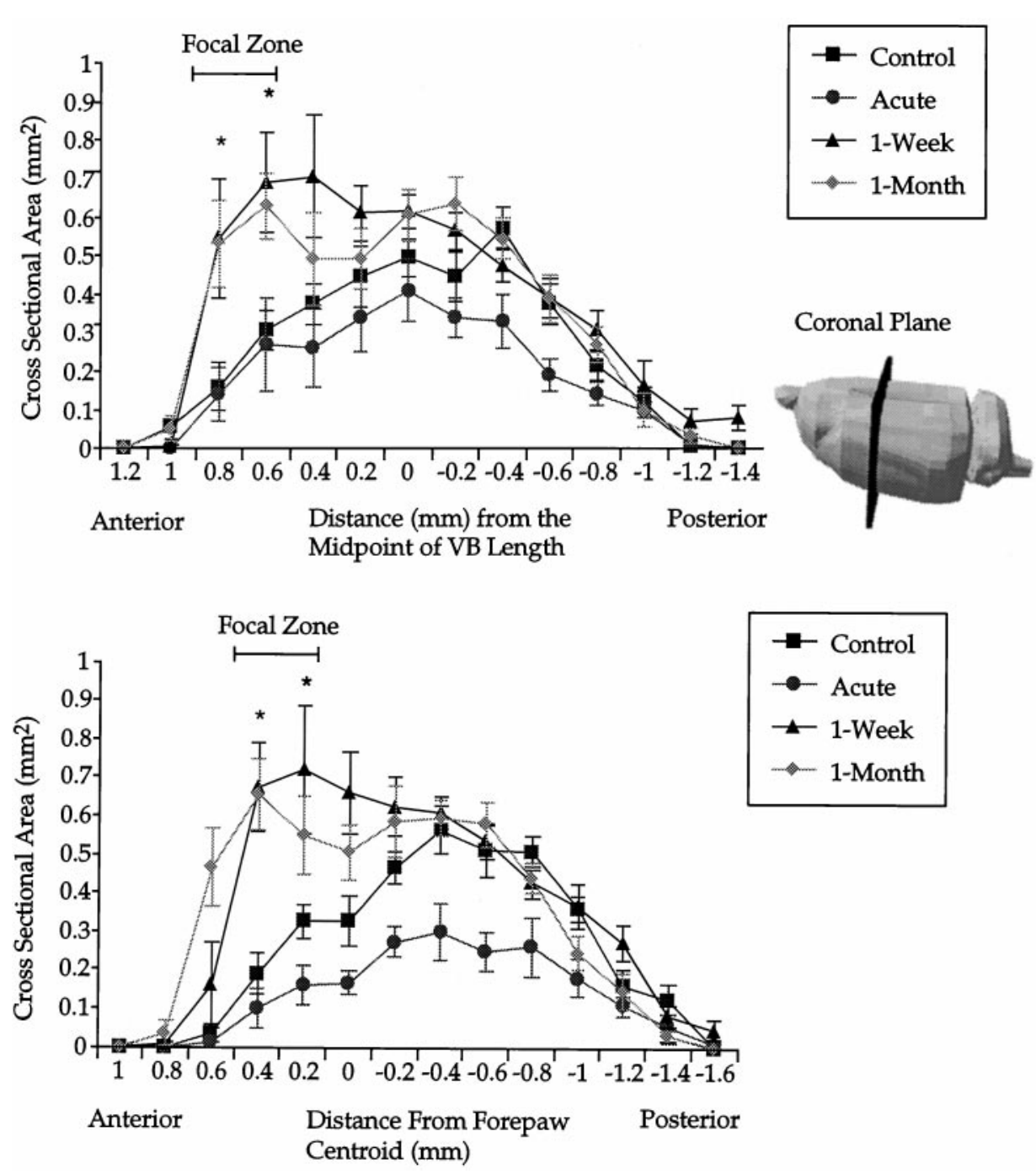

midpoint, whereas positive distances are anterior. Using this alignment, the average coronal area of all slices from animals at a given distance from the VB midpoint within a treatment group was obtained.

The increases in coronal area at 1 week and 1 month after lesion occurred almost exclusively at the rostral pole of the shoulder sensory map (Fig. $3 A$ ). A two-factor repeated measures ANOVA of group by distance revealed a significant effect of $\operatorname{group}\left(F_{(3,26)}=10.4 ; p<0.001\right)$ and distance $\left(F_{(6.47,168.2)}=41.3\right.$; $p<0.001)$ and an interaction $\left(F_{(19.41,168.2)}=2.9 ; p<0.001\right)$. Post hoc $t$ test comparisons revealed that both 1 week and 1 month postlesion groups at 0.6 and $0.8 \mathrm{~mm}$ from the VB midpoint were significantly different from the control group. At a rostral distance of $0.4 \mathrm{~mm}$, a post hoc $t$ test comparison between 1 week postlesion and control was significant, but 1 month postlesion versus control was not. Post hoc $t$ test comparisons between control and 1 week or 1 month postlesion were not significant at any other distance. The acute group displays a trend toward decreased area at almost all distances from the VB midpoint compared with controls, but with post hoc $t$ tests this was only significant at a distance of -0.6 $\mathrm{mm}$ relative to $\mathrm{VB}$.

We conducted the same type of comparisons in the coronal plane again with one difference; the identity of a coronal slice and the alignment of shoulder sensory maps was achieved by refer-
Figure 3. A, Coronal plane area of the shoulder sensory map as a function of anteroposterior distance. Maps from each animal are aligned with respect to the anatomically determined anteroposterior midpoint of the VB (anatomical registration). Lateral view of a rat brain indicates the plane of sectioning used on functional shoulder maps. The bar indicates a focal zone of robust change common to both 1 week and 1 month postlesion groups. Asterisks indicate that both 1 week and 1 month postlesion groups are significantly different from controls. Error bars indicate \pm SE. $B$, Coronal plane area of the shoulder sensory map as a function of the anteroposterior distance. Maps from each animal are aligned with respect to the centroid of the forepaw (functional registration). Asterisks indicate that both 1 week and 1 month postlesioned groups are significantly different from controls. Compared with anatomical alignment of coronal slices $(A)$, differences emerge more clearly with the functional alignment. All subsequent planar analysis is on coronal slices corresponding to the focal zone. Error bars indicate \pm SE. ence to the centroid of the forepaw representation (Fig. 3B). Because it appears that the forepaw representation is unaffected by nucleus gracilis lesions at the time intervals under inspection, we used its centroid as an alternative reference point.

For each animal, slices of the shoulder sensory map were identified by virtue of their anteroposterior distance from the centroid of the forepaw (Fig. 3B). For each animal within a treatment group, coronal slices of the same distance from the forepaw centroid were averaged. These coronal areas were plotted as a function of their distance from the forepaw centroid. With functional alignment, differences in this graph are consistent with differences detected with neuroanatomical alignment (Fig. $3 A$ ) but emerged more clearly.

A two-factor repeated measures ANOVA of the data depicted in Figure $3 B$ revealed a significant effect of group $\left(F_{(3,26)}=9.8\right.$; $p<0.001)$ and distance $\left(F_{(7.6,196.5)}=47.4 ; p<0.001\right)$ and an interaction $\left(F_{(22.7,196.5)}=4.5 ; p<0.001\right)$. When 1 week and 1 month postlesion groups were compared with controls, they were significantly larger at distances of 0.2 and $0.4 \mathrm{~mm}$, respectively, rostral to the forepaw centroid (post hoc $t$ tests). At a distance of $0.6 \mathrm{~mm}$, the 1 month postlesion group was significantly different from control, whereas the 1 week postlesion group was not (post hoc $t$ test). At the midpoint, only the 1 week postlesion group was significantly different from controls (post hoc $t$ test). Finally, 


\section{Control}

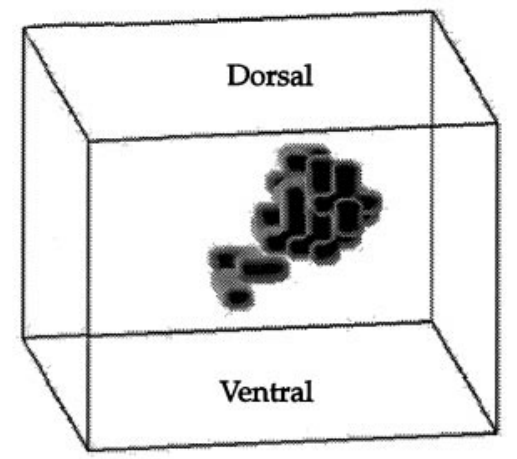

\section{Lesioned}

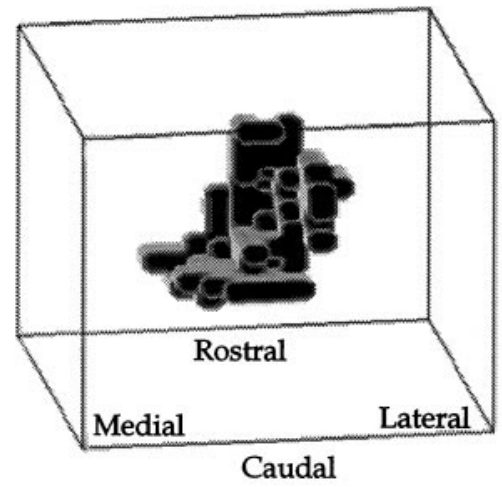

Figure 4. Spatial distribution of recording sites responsive to shoulder stimulation from control and 1 week postlesion animals. This is a caudal perspective of the sensory map. Lesion-induced changes are most marked at the rostral pole, toward the anterior end of the display. Much of the shoulder expansion appears to occur along the mediolateral axis after gracilis lesions.

significant differences emerged between acute and control groups from $0.2 \mathrm{~mm}$ rostral to $1.0 \mathrm{~mm}$ caudal to the centroid (except at the midpoint, with post hoc $t$ tests). At no point was there a significant difference between 1 week and 1 month postlesion (post hoc $t$ tests).

Displayed in Figure 4 are data from a control and 1 week postlesion animal for the shoulder sensory map. Each cube represents a recording site that was responsive to shoulder stimulation. The control map may on first inspection appear as two separate representations. However, minor discontinuities in sensory maps were not uncommon across animals and did not display any spatial consistency in their manifestation. If one averaged representations across animals, the composite map would appear solid in its entirety.

Functional alignment generated the same pattern of results as neuroanatomical registration of coronal slices, but differences appeared clearer. For this reason, and the relative ease with which a functional reference point permits comparisons in other planes, we used functional alignment for the remainder of the analysis. The region of interest with focal changes common to both 1 week and 1 month postlesion occurred at distances of 0.2 and $0.4 \mathrm{~mm}$, respectively, rostral to the forepaw centroid (Fig.

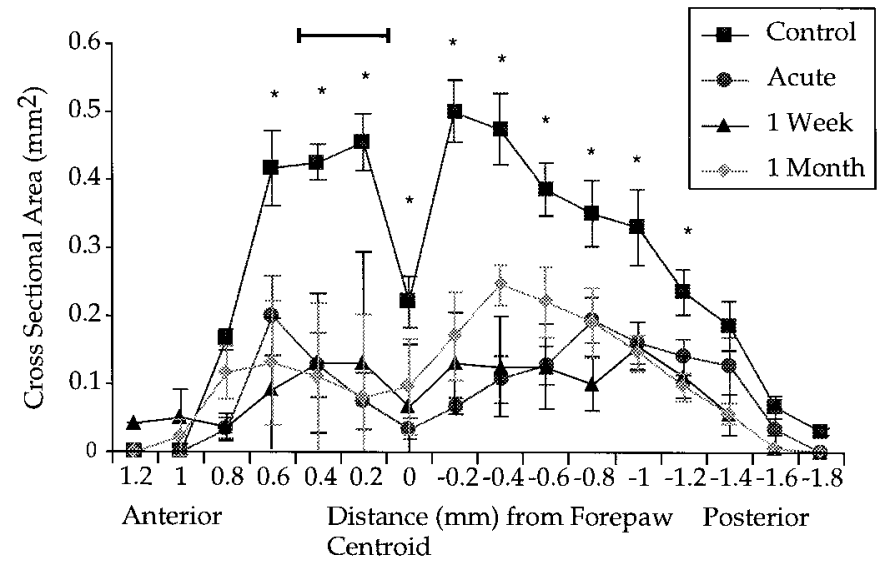

Figure 5. Plot of residual hindlimb input as a function of the anteroposterior axis. Abscissa coordinates are the same as those in Figure $3 B$. The area of hindlimb input corresponding to the focal zone relative to the forepaw centroid is indicated by the bar. Asterisks indicate significant differences between all treatment groups compared with control values. Error bars indicate $\pm \mathrm{SE}$.

$3 B)$. We identify this region of interest as a focal zone of reorganization.

Perhaps, however, our focal zone of expansion of the shoulder representation near the rostral pole is related to a massive loss of hindlimb input compared with more caudal segments of the map. In Figure 5, hindlimb data are plotted in the same manner as in Figure $3 B$ using functional alignment. An extensive loss of hindlimb input occurred in regions rostral and caudal to the forepaw centroid.

Statistical comparisons confirmed the extensive loss of hindlimb input rostrally and caudally to the forepaw centroid (Fig. 5). A two-factor repeated measures ANOVA revealed a main effect of group $\left(F_{(3,26)}=32.9 ; p<0.001\right)$ and distance $\left(F_{(9.1,237)}=28.2\right.$; $p<0.001)$ and an interaction $\left(F_{(27.3,237)}=5.5 ; p<0.001\right)$. The interaction suggests that the loss of hindlimb input was not constant with distance from the centroid. This result is largely attributable to the fact that relatively more hindlimb input remained intact at the caudal part of the map. The loss of hindlimb input at distances corresponding to the focal zone ( 0.2 and 0.4 $\mathrm{mm}$ anterior to centroid; Fig. 5, bar) was similar to that observed (e.g., $>60 \%$ loss) at more caudal distances of -0.2 to $-0.6 \mathrm{~mm}$. Yet, at these caudal positions, from -0.2 to $-0.6 \mathrm{~mm}$, extensive loss of hindlimb responses did not produce an expansion of the shoulder sensory map at corresponding coronal planes (as in Fig. $3 B$ ). Overall, post hoc $t$ test comparisons revealed a significant reduction of hindlimb input for all groups from 0.6 to $-1.2 \mathrm{~mm}$.

We asked how strong a predictor the reduction of hindlimb input would be of the ensuing shoulder expansion in the designated focal zone. We extracted from the functional shoulder map of each animal the two coronal slices corresponding to our focal zone of expansion $(0.2$ and $0.4 \mathrm{~mm}$ from the forepaw centroid; refer to Fig. $3 B$ ). For each animal we averaged the counts from these two slices and in turn averaged those values across animals within a group.

We looked at whether residual hindlimb volume in this focal zone $(0.2-0.4 \mathrm{~mm})$ could predict the degree of shoulder expansion. A linear regression, which combined 1 week and 1 month postlesion focal zones of each rat into a group large enough for this analysis, failed to reach significance $\left(F_{(1,14)}=3.6 ; p>0.08\right)$. Repeating the same analysis by looking at residual hindlimb input 


\section{Control}

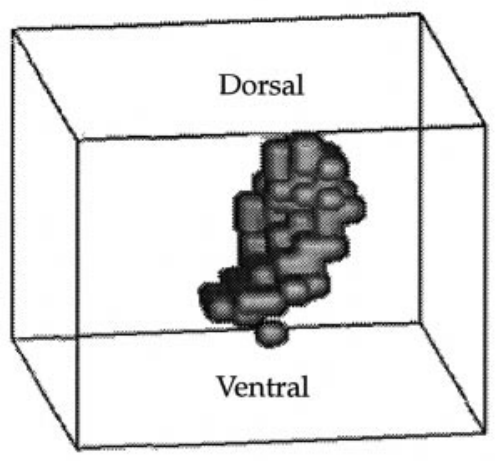

\section{Lesioned}

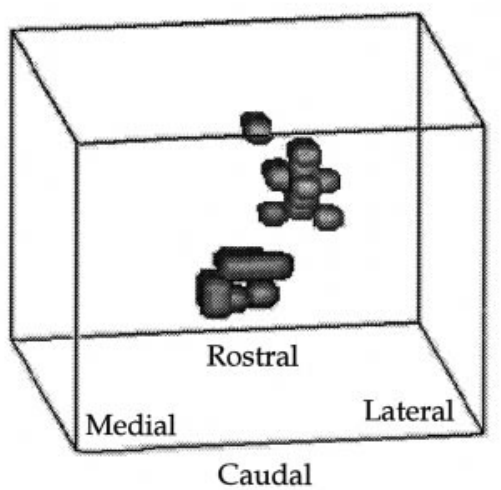

Caudal

Figure 6. Control and 1 week postlesioned animals. Spatial distribution of recording sites that were responsive to hindlimb stimulation are depicted from the caudal vantage point.

in the entire functional map as a predictor of shoulder expansion in the focal zone also failed to reach significance $\left(F_{(1,14)}=0.35\right.$; $p>0.5)$. Thus, the level of hindlimb loss did not predict the extent of shoulder expansion.

The spatial distribution of recording sites that were responsive to hindlimb stimulation is depicted in Figure 6. The intact hindlimb map in the control is restricted along the mediolateral axis but extends some distance in the dorsoventral direction. The extant hindlimb representation after a gracilis lesion indicated a number of punctate zones in which input appears to have remained intact, but overall the map is considerably reduced $(>60 \%)$ in size.

For the remainder of the planar analysis we continued to analyze the two coronal slices corresponding to the focal zone of shoulder expansion at 0.2 and $0.4 \mathrm{~mm}$ (Fig. $3 B$ ). Figure 7 depicts a profile of sagittal slices of the shoulder representation in this focal zone, taken along the mediolateral axis. Sample mapping data from a control animal from which Figure 6 was derived are displayed in Figure 1. The coronal slice taken in Figure 1 was taken at a distance from the forepaw centroid corresponding to the focal zone seen in lesioned animals. The identified unit corresponding to the centroid of the forepaw is enclosed in a box. To the left of the centroid (medially) are forepaw recording sites, and to the right of the centroid are shoulder and hindlimb recording sites.
Visual inspection of Figure 7 suggests that expansion of the shoulder representation at 1 week and 1 month after lesion progressed medially with reference to the forepaw centroid. A two-factor repeated measure ANOVA revealed a significant effect of group $\left(F_{(3,26)}=16.4 ; p<0.001\right)$ and distance $\left(F_{(6,155.5)}=\right.$ $39.7 ; p<0.001)$ and an interaction $\left(F_{(17.9,155.5)}=4.1 ; p<0.001\right)$. Compared with controls, 1 week and 1 month postlesion groups were significantly elevated in zones progressing from the centroid (zero) to $0.4 \mathrm{~mm}$ medially (post hoc $t$ tests). At $0.6 \mathrm{~mm}$ the 1 month postlesion group was significantly increased (post hoc $t$ test). The acute group did not reveal any significant contraction or expansion ( post hoc $t$ tests). It appears that along the mediolateral axis, expansion of the shoulder sensory map extended its border up to $0.6 \mathrm{~mm}$ medially. The small lateral expansion to the right of the forepaw centroid appears to be confined within the zone of the forepaw representation (Fig. 1).

In the horizontal plane, changes in cross-sectional area along the dorsoventral axis reveal that expansion occurs essentially at all depths in the focal zone (Fig. 8). This analysis examined the same two slices containing the focal zone that were scrutinized in the sagittal plane described above. A two-factor repeated measure ANOVA revealed a main effect of group $\left(F_{(3,26)}=11.2 ; p<\right.$ $0.001)$ and distance $\left(F_{(5.9,154.4)}=29.1 ; p<0.001\right)$ and an interaction $\left(F_{(17.8,154.4)}=4.8 ; p<0.001\right)$. Both 1 week and 1 month postlesion groups were significantly elevated compared with controls in the majority of horizontal planes, from $0.4 \mathrm{~mm}$ dorsal to the centroid to $-0.4 \mathrm{~mm}$ ventral (post hoc $t$ tests). At $0.6 \mathrm{~mm}$ dorsal to the centroid, 1 week postlesion was significant, whereas 1 month postlesion was not (post hoc $t$ tests). There was no significant difference between 1 week and 1 month postlesion at any horizontal level (post hoc $t$ tests). With regard to the acute group, there was a significant depression at the centroid when compared with controls (post hoc $t$ test).

\section{Histology}

Figure 9 is a photomicrograph of the dorsal column nuclei 1 week after lesion and shows that the damage to the gracile is very extensive. The cuneate nucleus on the right is very close to the medial edge of the left gracile nucleus with a small bar of gliotic tissue in between. Such damage was present along the length of the nucleus. There was also some suggestion of gliosis in the cuneate nucleus, although this appeared weak.

Figure 10 presents a photomicrograph of a Nissl-stained section of the thalamus in the horizontal plane of a lesioned animal (1 month after lesion). Sectioning the brains this way allowed us to visualize the entire pattern of electrode penetrations. Because the VPL is crescent-shaped in the coronal plane, penetrations at the presented slice level that appear too medial actually contact the VPL at more ventral levels. Consistently, our shoulder expansion appeared to occur at medial electrode penetrations at the rostral pole of the VPL.

\section{DISCUSSION}

Volumetric analysis indicated that expansion of the forelimb representation was confined to the shoulder region. However, such expansion in sensory map volume observed at 1 week and 1 month after lesion occurred after an unexpected transient contraction in volume at an acute time interval. Measures of representational overlap between forepaw and shoulder demonstrated the same temporal changes in volume as the shoulder sensory map. This latter data set suggested that the shoulder expanded by 
Figure 7. Cross-sectional area profile of shoulder representation. The focal zone is represented as a dark band in the coronal plane, which was cut sagittally with reference to the intact rat brain. Asterisks indicate significant differences between control versus 1 week and 1 month groups after lesion. As far as $0.6 \mathrm{~mm}$ medial to the forepaw centroid, 1 month postlesion was significantly elevated compared with control. Error bars indicate \pm SE.

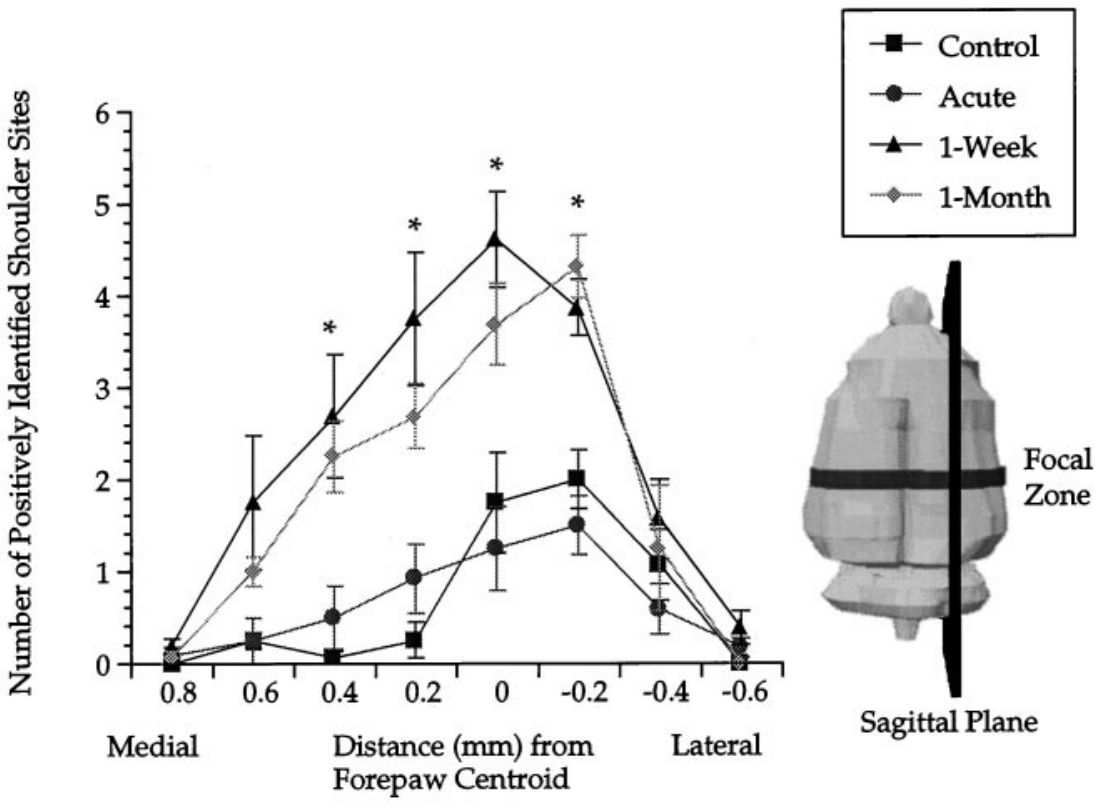

Figure 8. Profile of shoulder sensory representation, revealed by crosssectional slices in the horizontal plane. The focal zone is indicated as a dark coronal band, sectioned with reference to the intact rat brain. Asterisks indicate significant differences for both 1 week and 1 month postlesion groups versus control. Error bars indicate \pm SE.

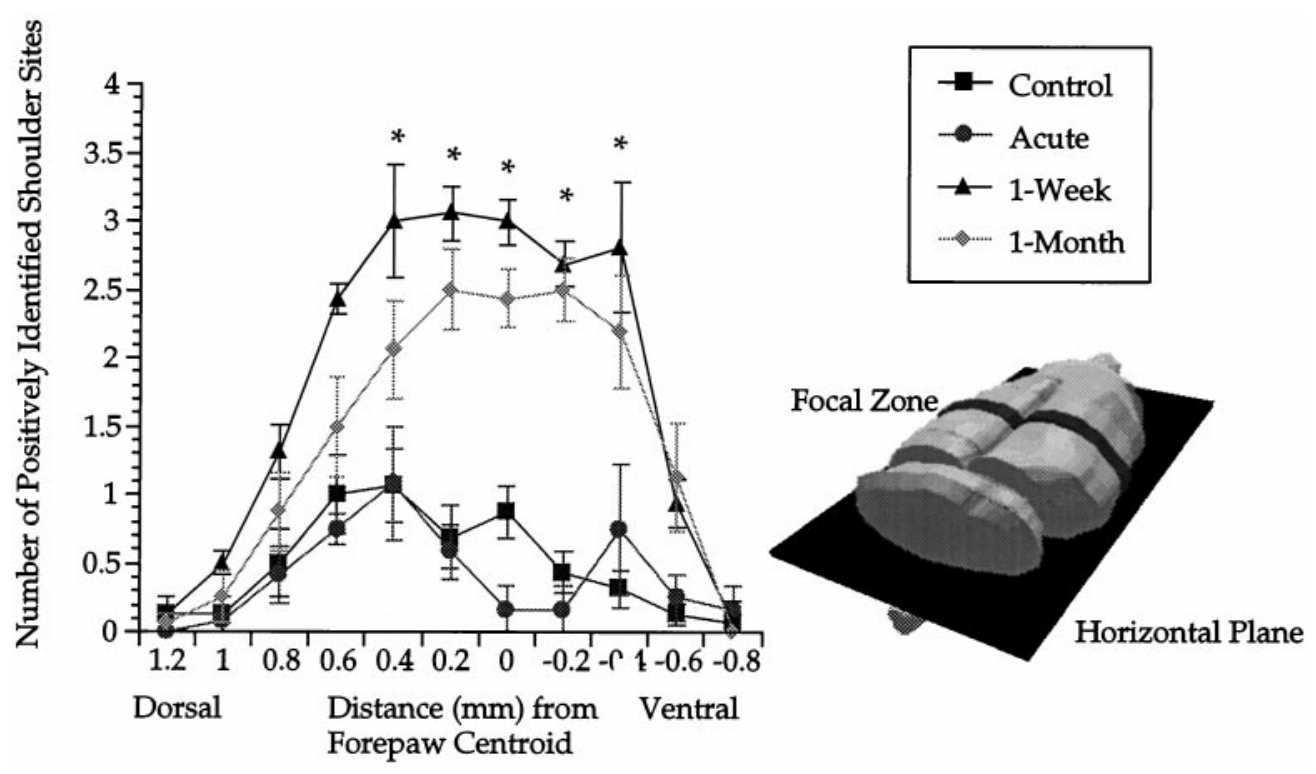

overlapping the forepaw representation, rather than expanding laterally into the denervated hindlimb zone.

Thalamic representation changes illustrated by volumetric data presaged similar findings in a detailed topological analysis. Remarkably, a focal zone of shoulder expansion was common at both 1 week and 1 month after lesion at $\sim 0.2-0.4 \mathrm{~mm}$ rostral to the forepaw centroid. Surprisingly, analysis of this focal zone in the sagittal plane clearly indicated that expansion progressed medially, overlaying the forepaw representation. As detailed in Results, there was no gradient or zone of hindlimb denervation that corresponded uniquely to our focal zone of shoulder expansion.

A schematic summary of the somatotopic changes occurring in our identified focal zone at 1 week and 1 month after lesion is presented (Fig. 11). The medial progression of the shoulder forepaw sensory map is derived from the volumetric data of compartmental overlap between shoulder and forepaw representations that increased after an acute time interval (Fig. 2). Addi- tionally, when the focal zone was sectioned in the sagittal plane (Fig. 7) as described above, a clear medial progression of the shoulder sensory map into the forepaw representation was revealed. Qualitatively this is consistent with what we observed in our raw data from the focal zone (Fig. 1B).

It is unlikely that our results are a product of the type of anesthetic used or subcortical bleeding. All groups were exposed to the same mapping protocol. Therefore, such an explanation fails to explain the different results obtained for each time point with respect to control. Furthermore, such an artifact is not consistent with our observed changes localized to the rostral pole of the VPL, rather than occurring throughout the length of the nucleus. Finally, since the acute group showed the opposite changes to later time points also suggests that our results are not the product of mapping-induced neuronal injury or anesthesia.

It was not necessary to control for possible morphological differences in the size of thalamic nuclei across animals. Although such differences may exist, there is no reason to believe that there 

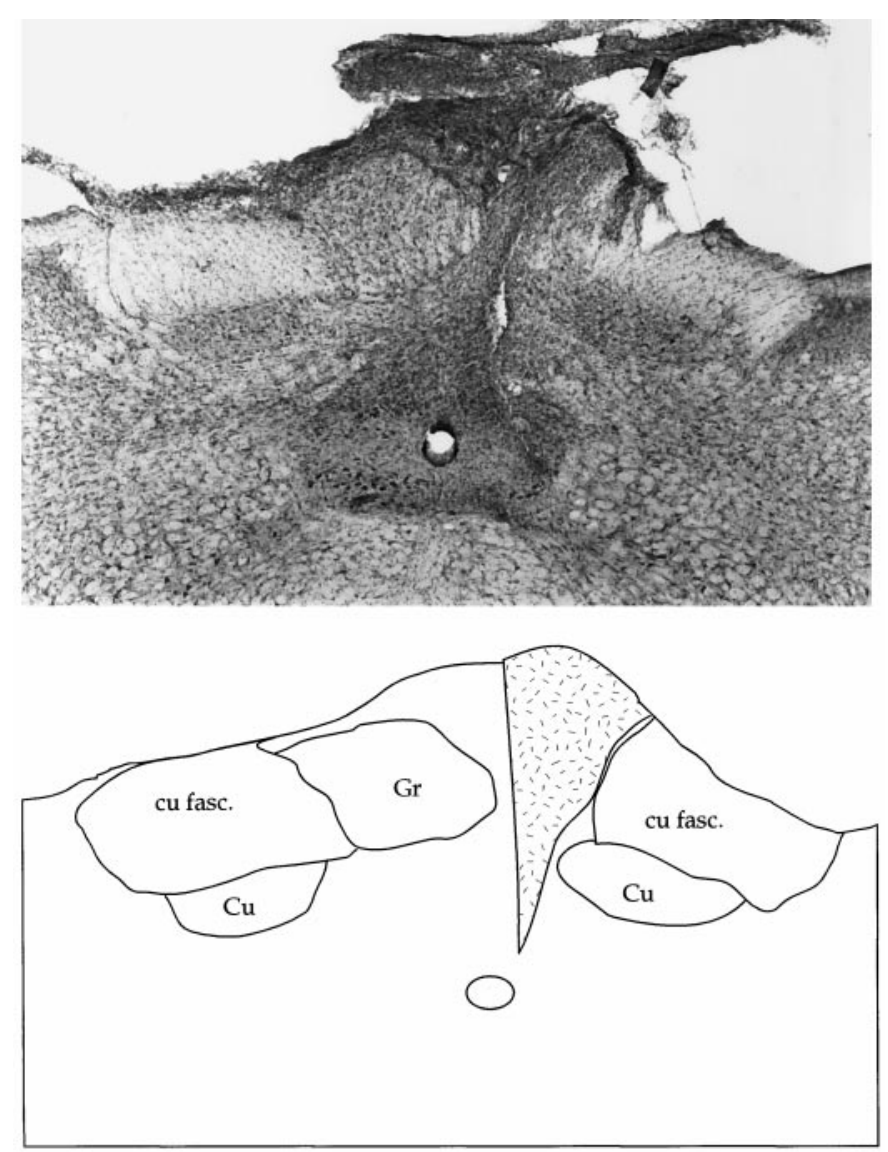

Figure 9. Photomicrograph depicting a coronal view of the dorsal column nuclei stained with Nissl 1 week after lesion. The damage observed in the gracile nucleus existed along the length of the nucleus. The accompanying diagram is a schematic rendering of the photomicrograph, barring tissue-processing artifacts. The zone of gliosis is indicated by the stippled area. cu fasc, Cuneatus fasciculus; $\mathrm{Cu}$, cuneate nucleus; $\mathrm{Gr}$, gracile nucleus.

are systematic differences between groups. If one argued for lesion-induced atrophy in the size of thalamic nuclei producing such differences, one would have to postulate increases in size of the VPL, because the absolute dimensions of the shoulder sensory map enlarged with time.

It may be possible that damage was caused to the shoulder input, as reflected in the contraction in its sensory map volume at an acute time interval. Histology did reveal some gliosis in the nucleus cuneatus. However, no statistically significant difference was seen between control and any of the lesioned groups for the forepaw sensory map volume. One would have expected damage to the forepaw sensory map if this hypothesis was correct, because it is interposed between the gracile nucleus and the shoulder functional map (Nord, 1966). Additionally, coronal slices of the shoulder sensory map outside of the focal zone at 1 and 4 weeks after lesion have exactly the same cross-sectional area as controls, suggesting that the contraction observed at an acute time interval is not a product of damage.

We have recently completed a preliminary study of the thalamus after nerve transection at 1 week after lesion (J. L. Parker and J. O. Dostrovsky, unpublished data). We have observed changes after nerve transection similar to those described in this study. This suggests that possible damage to nucleus cuneatus caused by gracile lesions or residual hindlimb input to VPL is not

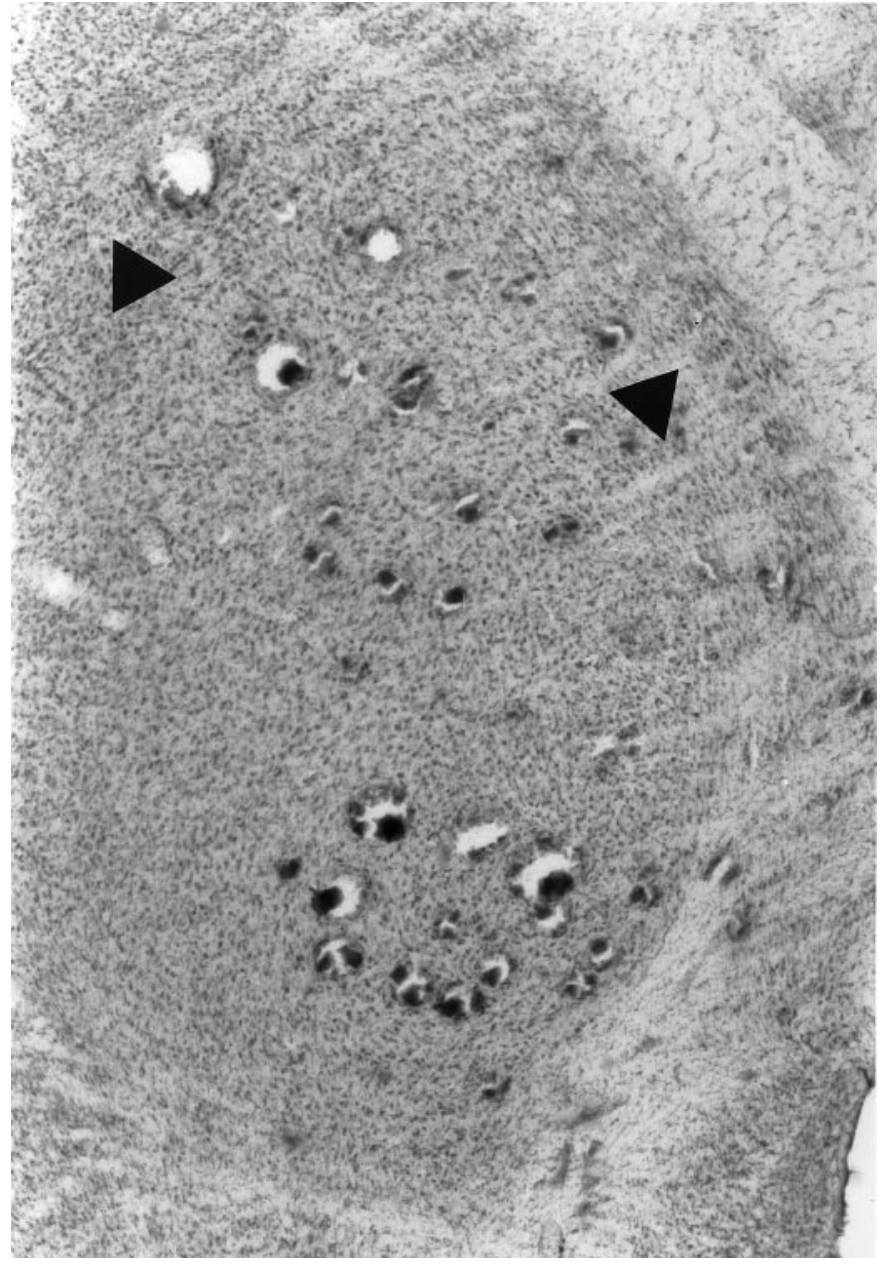

Figure 10. Photomicrograph of a 1 month postlesion rat brain sectioned in the horizontal plane and stained with cresyl violet. Arrows are interposed between two rows of electrode penetrations corresponding to the focal zone.

a likely explanation for the pattern of reorganization described herein.

Concordant with the results of Wall and Egger (1971), our lesions of nucleus gracilis failed to block hindlimb input completely. It is likely that the extant hindlimb sensory map was receiving input from pathways independent of the dorsal column nuclei. The spinocervical tract can convey cutaneous information and does so independently of the dorsal column nuclei (Morin, 1955; Giesler et al., 1979). In addition, in the rat it appears that many spinothalamic tract neurons respond to innocuous inputs, and at least some of these neurons terminate in the VPL (Dado et al., 1994). Either one of these pathways may have been responsible for mediating the residual hindlimb input observed in this study.

There were also clear differences in this report from that of Wall and Egger (1971). The most poignant difference is that shoulder expansion did not appear to progress into the denervated hindlimb zone but rather annexed territory by superimposing itself on the existing forepaw representation. Importantly, this change was contained in a $300 \mu \mathrm{m}$ coronal slice toward the rostral pole of the VPL. Such discrepancies may be related to differences in the choice of the control group. Wall and Egger (1971) used the hemisphere ipsilateral to their gracile lesion as a within-subject 


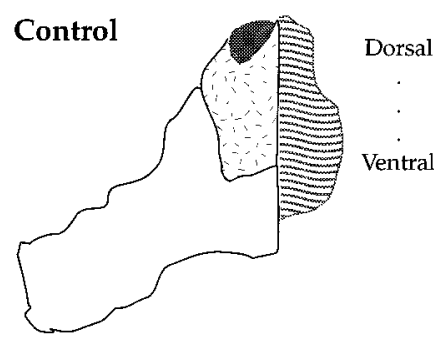

Medial ... Lateral

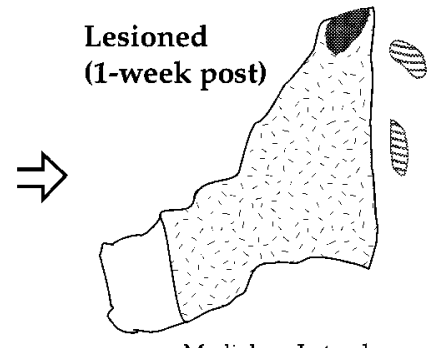

Medial ... Lateral

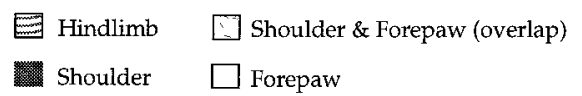

Figure 11. Schematic of the topographic overlap between shoulder and forepaw in the focal zone at 1 week and 1 month after lesion. This is a coronal view of forepaw, shoulder, and hindlimb sensory maps in the VPL. The plasticity depicted is derived from data on the volume of shoulder-forepaw overlap (Fig. 2) and planar analysis (Figs. 7, 8).

control. However, we now know that there are ipsilateral consequences from a lesion (before its decussation), such as somatotopic plasticity (Calford and Tweedale, 1990) and changes in acetylcholine binding in the cortex (Hanisch et al., 1992). In comparing our treatment groups against a separate group of nonlesioned animals, we have avoided possible misinterpretation in using the contralateral hemisphere as a control.

One mechanism that may account for the contraction of the shoulder functional volume is the emergence of inhibitory receptive fields, which are known to result from deafferentation (Dostrovsky et al., 1976; Rasmusson et al., 1993). After digit amputation in the raccoon, neurons that have suffered loss of input acquire inhibitory receptive fields on adjacent intact digits ("off-focus inhibition"), which depress spontaneous activity when stimulated (Northgrave and Rasmusson, 1996). Consistent with the transient character of our shoulder map volume contraction, inhibitory receptive fields are observable at acute time intervals but appear to be absent in chronic preparations (Dostrovsky et al., 1976; Northgrave and Rasmusson, 1996; Rasmusson, 1996b).

A decrease in shoulder map volume at an acute time interval may be the product of off-focus inhibition by neurons that previously had receptive fields for shoulder and hindlimb. Single-unit characterization of VPL neurons has encountered neurons with large receptive fields that can cover virtually half of the body surface of the rat (Sherman et al., 1998). In mapping acutely lesioned animals, off-focus inhibition generated by a few neurons with receptive fields covering the shoulder and hindlimb would cause a decrease in spontaneous activity of other neurons at the recording site. Accordingly, reduced background activity attenuates the discharge probability of other neurons that are responsive to shoulder stimulation. Off-focus inhibition generated by a few units at a recording site reduces the number of recording sites responsive to shoulder stimulation, which in our paradigm is reflected as an overall decrease in the shoulder map volume.

An overview of the literature might suggest that the direction of our shoulder expansion is inconsistent with the maxim that increased somatotopic representation occurs via border extensions into an adjacent deafferented zone (Wall and Egger, 1971; Kalaska and Pomeranz, 1979; Merzenich et al., 1983, 1987; Wall and Cusick, 1984). However, there may be an unusual relationship between forelimb and hindlimb somatotopic maps that may warrant the suspension of this maxim in this case. Research at the cortical level that has examined changes in somatotopy after fasciculus gracilis lesions failed to find any expansion of the forelimb into hindlimb (Jaine et al., 1995). Investigations with optical imaging of the cerebral cortex in rats have found considerable topographic overlap between different skin regions within either the forelimb or hindlimb (Godde et al., 1995). In contrast, Godde et al. (1995) found no overlap between the forelimb and hindlimb representations, leading to the speculation that these two somatotopic domains may be functionally insulated from each other in the context of plasticity. One might argue that cortical and thalamic somatotopic changes may be independent (Wang et al., 1995) or question whether somatotopic territory can be exchanged between hindlimb and forelimb.

If we accept provisionally that border shifts between forelimb and hindlimb representations do not occur, it is not immediately obvious why the loss of hindlimb input would induce topographic overlap between shoulder and forepaw. There is good evidence supporting the view (Merrill and Wall, 1972) that inhibition is important in restricting receptive field size, and this has been confirmed at the cortical (Hicks and Dykes, 1983; Dykes et al., 1984) and thalamic levels (Lee et al., 1994). Interestingly, recordings from the VPL revealed mutual inhibition between forelimb and hindlimb units in $45 \%$ of the neurons tested in response to electrical stimulation (Roberts and Wells, 1990). Perhaps the loss of the hindlimb representation removed inhibition acting on units in the forepaw representation of the VPL, leading to an increase in their receptive field size, enabling these units to respond to shoulder stimulation.

\section{Hot spot model}

The plastic focal zone we have described in the thalamus after nucleus gracilis lesions appears to have four properties: (1) it is a subregion of a sensory map defined by low-threshold mechanical input that appears to display somatotopic reorganization; (2) it reveals reorganizational events that occur over chronic rather than acute time intervals; (3) reorganizational events take place in a zone that is a slice; and (4) this slice appears to be oriented orthogonally to columns of cells possessing the same receptive fields.

Property 4 is partly an inference drawn from the literature. Jones et al. (1979, 1982) and Jones and Friedman (1982) have built a persuasive case that the columnar organization of the cerebral cortex (Mountcastle, 1957) is also found in the VB of the thalamus. These "thalamic rods" are analogous to the columnar organization of the cortex, because within a rod cells possessed the same receptive field properties (Jones et al., 1982). Furthermore, these rods were proposed to be oriented along the anteroposterior axis in the thalamus. If thalamic rods exist, they intersect our slice of plasticity at approximately a $90^{\circ}$ angle (Fig. 12).

We speculate that this focal zone may be a hot spot that displays thalamic reorganization in general and may have manifestations elsewhere in the nervous system. This hot spot hypothesis has two predictions. First, the thalamic hot spot is not specific to nucleus gracilis lesions but may also be important in displaying somatotopic reorganization after other types of neural trauma (e.g., nerve transection) (Parker and Dostrovsky, unpublished data). Second, the existence of a hot spot of plasticity is not a feature specific to the thalamus but may also exist in other structures such as the cerebral cortex or dorsal column nuclei.

If a hot spot exists in the cerebral cortex, properties 3 and 4 would predict the orientation of the slice that reveals reorganization. The existence of columns of cells with isomorphic receptive 

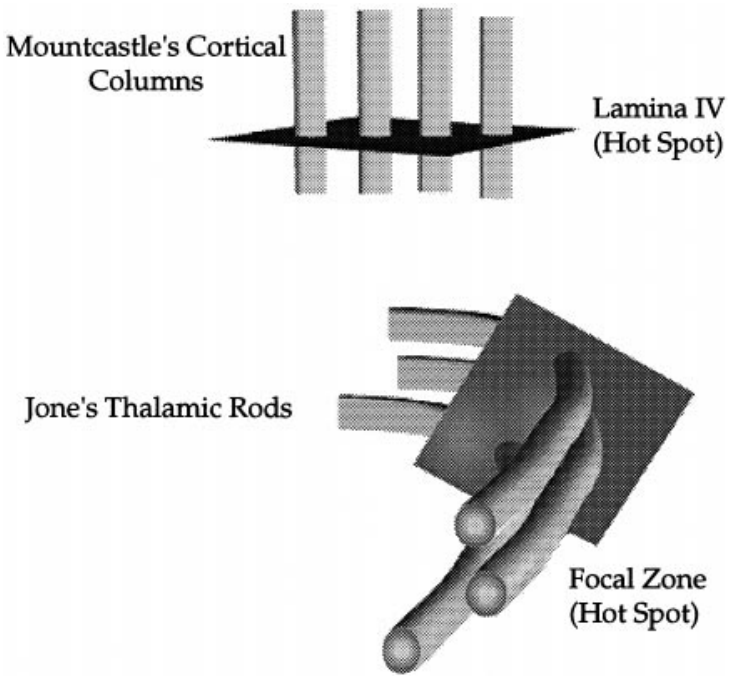

Figure 12. Hot spot model of neural plasticity. A depiction of our identified focal zone or hot spot of reorganization in the thalamus. It is proposed that hot spots exist at other levels of the somatosensory pathway such as the cortex. Cortically, the hot spot is a slice oriented orthogonally to the cortical columns of Mountcastle (1957), in the same way the thalamic hot spot is orthogonally oriented with respect to the thalamic rods of Jones et al. (1979, 1982) and Jones and Friedman (1982). The somatotopy of the thalamus is rotated compared with that of the cortex, given the difference in the orientation of thalamic rods and cortical columns. See Discussion.

field properties is organized vertically in the cortex (Mountcastle, 1957), opposite to the orientation of thalamic rods (Fig. 12). According to property 4 of our hot spot description, the slice would be oriented horizontally in the cortex, orthogonal to the cortical columns of Mountcastle (1957).

Properties 1 and 2 of the hot spot applied to the cortex would predict the existence of a circumscribed zone displaying plasticity over chronic time intervals. Although a consensus may not exist with respect to the role of each lamina in mediating plasiticity, it is clear that over time specific lamina seem to be particularly modifiable (Diamond et al., 1994; Kirkwood and Bear, 1994). In terms of the hot spot hypothesis, a slice displaying plasticity may include one or a subset of the six lamina in the cortex (property 1). For illustrative purposes we have suggested layer IV (Fig. 12), which seems to undergo plasticity over chronic rather than acute time intervals (Jenkins et al., 1990; Recanzone et al., 1992; Diamond et al., 1994), consistent with property 2 of the hot spot.

Differences in the orientation of hot spots at each level of the neuraxis can determine how easily they are detected with standard vertical electrode penetrations. Statistically, a large number of electrode penetrations would be required before the thalamic hot spot was detected, whereas in the cortex, every vertical electrode penetration traverses this plastic slice (Fig. 12). This difference may explain why learning-induced changes in cortical somatotopy were not detected in the thalamus (Wang et al., 1995). In our view, a detailed 3-D reconstruction of the entire somatotopic map in the thalamus would be necessary to detect such a zone.

In summary, the hot spot hypothesis can make predictions about the time course and orientation of a slice of plasticity at different levels of the nervous system. We do not venture to speculate on what the inter-relationships may be between hot spots at different levels of the neuraxis, if they in fact exist outside of the thalamus.

\section{Conclusions}

To our knowledge, the contraction of sensory maps at acute time intervals after lesion followed by increased topographic overlap represent new properties of thalamic plasticity. Furthermore, such topographic overlap mediating shoulder sensory map expansion occurred within a narrow coronal slice of the VPL that we have referred to as a focal zone. Thus, it appears that thalamic sensory maps defined by low-threshold mechanical input are not homogeneous with respect to their potential for plasticity.

\section{REFERENCES}

Alloway KD, Aaron GB (1996) Adaptive changes in the somatotopic properties of individual thalamic neurons immediately following microlesions in connected regions of the nucleus cuneatus. Synapse 22:1-14.

Basbaum AI, Wall PD (1976) Chronic changes in the response of cells in adult cat dorsal horn following partial deafferentation: the appearance of responding cells in a previously non-responsive region. Brain Res 116:181-204.

Calford MB, Tweedale R (1990) Interhemispheric transfer of plasticity in the rat cerebral cortex. Science 249:805-807.

Dado RJ, Katter JT, Giesler GJJ (1994) Spinothalamic and spinohypothalamic tract neurons in the cervical enlargement of rats. II. Responses to innocuous and noxious mechanical mechanical and thermal stimuli. J Neurophysiol 71:981-1002.

Devor M, Wall PD (1978) Reorganization of spinal cord sensory map after peripheral nerve injury. Nature 275:75-76.

Diamond ME, Huang W, Ebner FF (1994) Laminar comparison of somatosensory cortical plasticity. Science 265:1885-1888.

Dostrovsky JO, Millar J, Wall PD (1976) The immediate shift of afferent drive of dorsal column nucleus cells following deafferentation: a comparison of acute and chronic deafferentation in gracile nucleus and spinal cord. Exp Neurol 52:480-495.

Dykes RW, Landry P, Metherate RS, Hicks TP (1984) Functional role of GABA in cat primary somatosensory cortex: shaping receptive fields of cortical neurons. J Neurophysiol 52:1066-1093.

Fadiga E, Haimann C, Margnelli M, Sotgiu ML (1978) Variability of peripheral representation in ventrobasal thalamic nuclei of the cat: effects of acute reversible blockade of the dorsal column nuclei. Exp Neurol 60:484-498.

Garraghty PE, Kaas JH (1991) Functional reorganization in adult monkey thalamus after peripheral nerve injury. NeuroReport 2:747-750.

Giesler GJJ, Urca G, Cannon T, Liebeskind JC (1979) Response properties of neurons of the lateral cervical nucleus in the rat. J Comp Neurol 186:65-78.

Godde B, Hilger T, von Seelen W, Berkefeld T, Dinse HR (1995) Optical imaging of rat somatosensory cortex reveals representational overlap as a topographic principle. NeuroReport 7:24-28.

Hanisch U-K, Rothe T, Krohn K, Dykes RW (1992) Muscarinic cholinergic receptor binding in rat hindlimb somatosensory cortex following partial deafferentation by sciatic nerve transection. Neurochem Int 21:313-327.

Hicks TP, Dykes RW (1983) Receptive field size for certain neurons in primary somatosensory cortex is determined by GABA-mediated intracortical inhibition. Brain Res 274:160-164.

Jaine NJ, Florence SL, Kaas JH (1995) Limits on plasticity in somatosensory cortex of adult rats: hindlimb cortex is not reactivated after dorsal column transection. J Neurophysiol 73:1537-1546.

Jenkins WM, Merzenich MM, Ochs MT, Allard T, Guic-Robles E (1990) Functional reorganization of primary somatosensory cortex in adult owl monkeys after behaviorally controlled tactile stimulation. J Neurophysiol 63:82-104.

Jones EG, Friedman DP (1982) Projection pattern of functional components of thalamic ventrobasal complex on monkey somatosensory cortex. J Neurophysiol 48:521-544.

Jones EG, Wise SP, Coulter JD (1979) Differential thalamic relationships of sensory-motor and parietal cortical fields in monkeys. J Comp Neurol 183:833-882.

Jones EG, Friedman DP, Hendry HC (1982) Thalamic basis of placeand modality-specific columns in monkey somatosensory cortex: a correlative anatomical and physiological study. J Neurophysiol 48:545-568.

Kalaska J, Pomeranz B (1979) Chronic paw denervation causes an age- 
dependent appearance of novel responses from forearm in "paw cortex" of kittens and adult cats. J Neurophysiol 42:618-633.

Kirkwood A, Bear MF (1994) Hebbian synapses in visual cortex. J Neurosci 14:1634-1645.

Lee SM, Friedberg MH, Ebner FF (1994) The role of GABA-mediated inhibition in the rat ventral posterior medial thalamus. I. Assessment of receptive field changes following thalamic reticuler nucleus lesions. J Neurophysiol 71:1702-1715.

Merrill EG, Wall PD (1972) Factors forming the edge of a receptive field: the presence of relatively ineffective afferent terminals. J Physiol (Lond) 226:825-846.

Merzenich MM, Kaas JH, Wall JT, Nelson RJ, Sur M, Felleman DJ (1983) Progression of change following medial nerve section in the representation of the hand in area $3 \mathrm{~b}$ and 1 in adult owl and squirrel monkeys. Neuroscience 10:639-665.

Merzenich MM, Nelson RJ, Kaas JH, Stryker MP, Jenkins WM, Zook JM, Cynader MS, Schoppmann A (1987) Variability in hand surface representations in areas $3 \mathrm{~b}$ and 1 in adult owl and squirrel monkeys. J Comp Neurol 258:281-296.

Millar J, Basbaum AI, Wall PD (1976) Restructuring of the somatotopic map and appearance of abnormal neuronal activity in the gracile nucleus after partial deafferentation. Exp Neurol 50:658-672.

Morin F (1955) A new spinal pathway for cutaneous impulses. Am J Physiol 130:245-252.

Mouncastle, VB (1957) Modality and topographic properties of single neurons of cat's somatic sensory cortex. J Neurophysiol 20:408-434.

Nakahama H, Nishioka S, Otsuka T (1966) Excitation and inhibition in ventrobasal thalamic neurons before and after cutaneous input deprivation. Prog Brain Res 21A:180-185.

Nicolelis MAL, Lin RCS, Woodward DJ, Chapin JK (1993) Induction of immediate spatiotemporal changes in the thalamic networks by peripheral block of ascending cutaneous information. Nature 361:533-536.

Nord GN (1966) Somatotopic organization in the spinal trigeminal nucleus, the dorsal column nuclei and related structures in the rat. J Comp Neurol 130:343-356.

Northgrave SA, Rasmusson D (1996) The immediate effect of peripheral deafferentation on neurons of the cuneate nucleus in raccoons. Somatosens Mot Res 13:103-113.

Parker JL, Dostrovsky JO (1996) Spatial dynamics of thalamic plasticity. Can Physiol Soc 27:122.

Paxinos G, Watson C (1997) The rat brain in stereotaxic coordinates, Ed 3. New York: Academic.
Pollin B, Albe-Fessard D (1979) Organization of somatic thalamus in monkeys with and without section of dorsal spinal tracts. Brain Res 173:431-449.

Rasmusson D (1996a) Changes in the organization of the ventroposterior lateral thalamic nucleus after digit removal in adult raccoon. J Comp Neurol 364:93-103.

Rasmusson DD (1996b) Changes in the response properties of neurons in the ventroposterior lateral thalamic nucleus of the raccoon after peripheral deafferentation. J Neurophysiol 75:2441-2450.

Rasmusson DD, Louw DF, Northgrave SA (1993) The immediate effects of peripheral denervation on inhibitory mechanisms in the somatosensory thalamus. Somatosens Mot Res 10:69-80.

Recanzone GH, Merzenich MM, Jenkins WM, Grajski KA, Dinse HR (1992) Topographic reorganization of the hand representation in cortical area $3 \mathrm{~b}$ of owl monkeys trained in frequency-discrimination task. J Neurophysiol 67:1031-1056.

Rhoades RW, Belford GR, Killackey HP (1987) Receptive-field properties of rat ventral posterior medial neurons before and after selective kainic acid lesions of the trigeminal brainstem complex. J Neurophysiol 57:1577-1600.

Roberts WA, Wells J (1990) Extensive dual innervation and mutual inhibition by forelimb and hindlimb inputs to ventroposterolateral nucleus projection neurons in the rat. Somatosens Mot Res 7:85-95.

Sherman SE, Luo L, Dostrovsky JO (1998) Altered Receptive Fields and sensory modalities of rat VPL Thalamic neurons during spinal strychnine-induced allodynia. J Neurophysiol, in press.

Shin H-C, Park S, Son J, Sohn J-H (1995) Responses from new receptive fields of VPL neurones following deafferentation. NeuroReport 7:33-36.

Snow PJ, Wilson P (1991) Plasticity and somatosensory thalamus. In: Plasticity in the somatosensory system of developing and mature mammals - the effects of injury to the central and peripheral nervous system (Autrum H, Ottoson D, Perl ER, Schmidt RF, Shimazu H, Willis WD, eds), pp 286-311. Berlin: Springer.

Wall PD, Egger MD (1971) Formation of new connexions in adult rat brains after partial deafferentation. Nature 232:542-545.

Wall PT, Cusick CG (1984) Cutaneous responsiveness in primary somatosensory (S-1) hindpaw cortex before and after partial hindpaw deafferentation in adult rats. J Neurosci 4:1499-1515.

Wang X, Merzenich MM, Sameshima K, Jenkins WM (1995) Remodelling of hand representation in adult cortex determined by timing of tactile stimulation. Nature 378:71-75. 